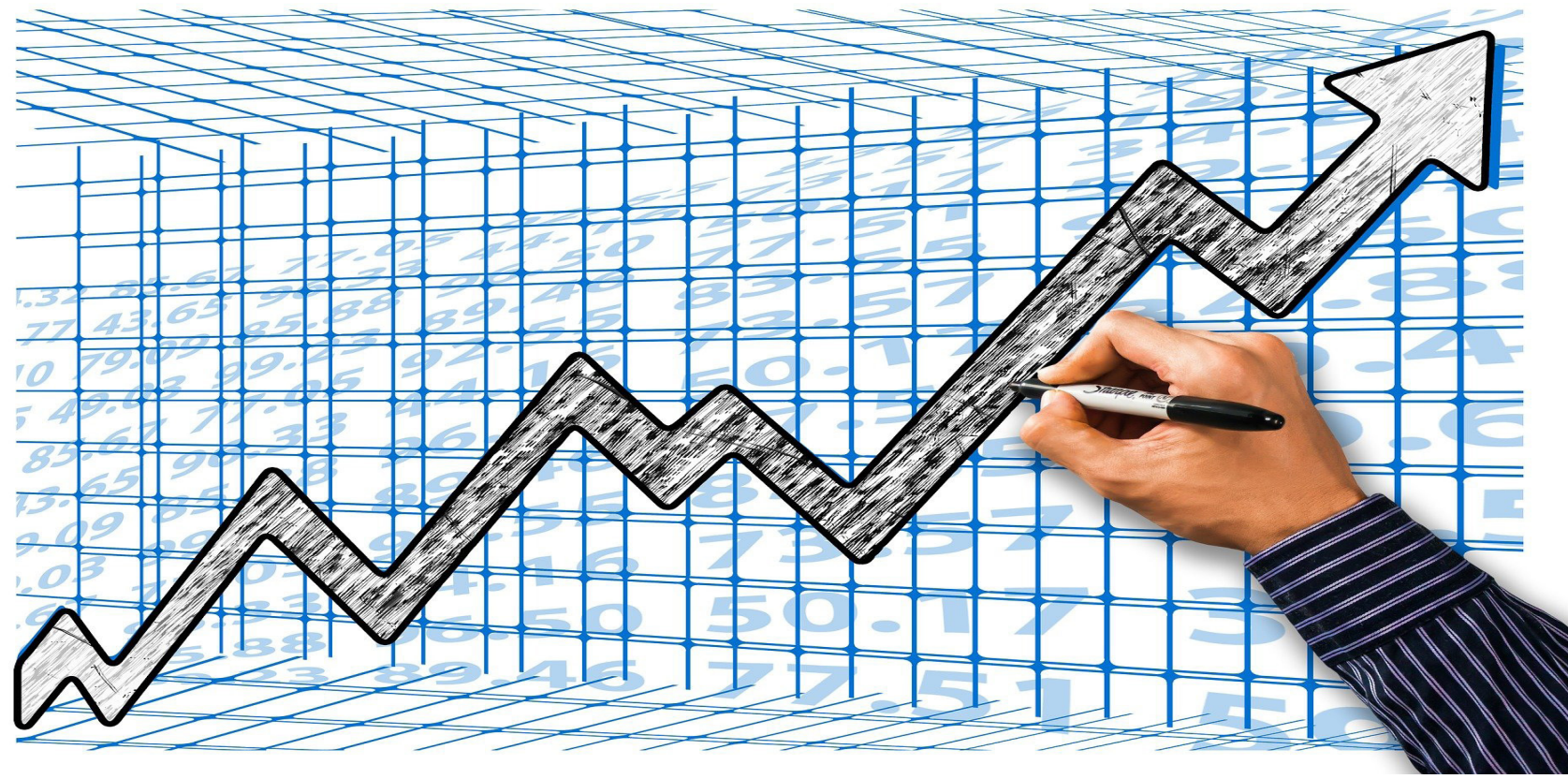

\title{
Aportes y limitaciones del análisis financiero que emplean las pequeñas y medianas empresas
}

\section{Contributions and limitations of the financial analysis used by small and medium-sized companies}

Ingrid Johanna Archila-Soto

Estudiantes del Programa de Contaduría Pública, Universidad Francisco de Paula Santander, Cúcuta, Colombia
Luis Fernando Guardiola-Plazas

Magister en Gerencia de Empresas Mención Finanzas, luisfernandogp@ufps.edu.co, Universidad Francisco de Paula Santander, Cúcuta, Colombia

Edwin Daniel Albarracín-Ortega

Estudiantes del Programa de Contaduría Pública,

Universidad Francisco de Paula Santander, Cúcuta,

Colombia 
Cómo citar: I.J. Archila-Soto, E.D.Albarracín-Ortega, L.F. Guardiola-Plazas. (2018). Aportes y limitaciones del análisis financiero que emplean las pequeñas y medianas empresas. Reflexiones contables (Cúcuta), 1 (1), 48-60.

\section{Resumen}

Este artículo trata sobre los aportes y limitaciones del análisis financiero que emplean en la actualidad las pequeñas y medianas empresas PYMES. La metodología consistió en una revisión documental, mediante trabajos extraídos de la base de datos Scopus y Dialnet de la biblioteca Eduardo Cote Lamus. Se consultaron 20 publicaciones relacionadas con el tema en un periodo de tiempo de los últimos 10 años, tanto a nivel internacional, como en el ámbito nacional. En los resultados se lograron identificar las principales herramientas de análisis financiero que pueden utilizar las PYMES en la actualidad. Seguidamente, dentro de las publicaciones consultadas se identificaron los principales problemas financieros que se reportaron, en PYMES que conforman diferentes sectores como el comercial, industrial y de servicios. A partir de esta información, se propusieron las herramientas que pueden mejorar la gestión en estas empresas para enfrentar los problemas de acceso a capital de trabajo, reducción de los costos financieros y para mejorar la práctica de planificación, de manera que les permita reducir la incertidumbre y el riesgo de pérdidas en el mercado cambiante.

Palabras clave: Herramientas Financieras, Pymes, Análisis Financiero, Capital de Trabajo.

\section{Abstract}

This article deals with the contributions and limitations of the financial analysis currently used by small and medium-sized SMEs. The methodology consisted of a documentary review, through works extracted from the Scopus and Dialnet databases of the Eduardo Cote Lamus library. Twenty publications related to the subject were consulted in a period of time of the last 10 years, both at the international level and at the national level. The results were able to identify the main financial analysis tools that SMEs can use today. Next, within the consulted publications, the main financial problems that were reported were identified, in SMEs that make up different sectors such as commercial, industrial and services. Based on this information, tools that can improve management in these companies were proposed to face problems of access to working capital, reduction of financial costs and to improve planning practice, in a way that allows them to reduce uncertainty. and the risk of losses in the changing market.

Keywords: Financial Tools, SMEs, Financial Analysis, Working Capital. 


\section{0}

\section{Introducción}

Debido a su importante papel en la creación de nuevos puestos de trabajo, el aumento del PIB, el espíritu empresarial y la innovación, se reconoce que las pequeñas y medianas empresas (PYME) son los motores del crecimiento socioeconómico, tanto en las economías desarrolladas, como en las de países en vías de desarrollo. En Colombia, el 99\% de las PYME, según cifras del Ministerio de Comercio, Industria y Turismo (citado por Barbosa, Camelo, Deaza \& Rodríguez, 2018), generan cerca del $40 \%$ del Producto Interno Bruto (PIB) y contribuyen con el $80 \%$ del empleo del país.

Por lo tanto, la importancia de las PYME para la economía y la sociedad es mucho mayor, en comparación con otros países emergentes y desarrollados. Estas, se enfrentan a una serie de desafíos, mientras que los problemas derivados de la "mala gestión financiera" se consideran las principales causas de quiebras comerciales en las PYME. La Gestión Financiera Estratégica, es un área de investigación que ha atraído el interés de los investigadores a partir de 2016, siento la misma, una de las áreas clave de gestión de las PYMES, debido a su papel vital en la supervivencia, crecimiento y desempeño de la economía.

El propósito de este documento es analizar el papel central de la gestión financiera e identificar los aportes y limitaciones del análisis financiero que emplean las pequeñas y medianas empresas PYMES, desde una perspectiva de gestión estratégica. En el transcurso de este estudio, se identifican las principales herramientas de análisis en materia financiera que utilizan las pequeñas y medianas empresas. Para posteriormente, describir la situación en ese sentido que presentan las mismas y finalmente, proponer herramientas que fortalezcan la rentabilidad y el aprovechamiento de los activos en este grupo de empresas. Además, se espera que el marco conceptual desarrollado sea útil para los académicos en el desarrollo de una agenda para futuras investigaciones empíricas.

\section{Teoría de análisis financieros}

Debido a los grandes cambios presentados en el entorno empresarial, los gerentes tienen la necesidad de poseer un conocimiento de alto nivel que les permita tomar decisiones rápidas y pertinentes; estos requieren aplicar herramientas útiles para administrar sus negocios de manera eficiente y lograr los objetivos. En general, según Angulo, Berrio \& Caicedo (2014), las organizaciones cuando tienen problemas financieros, como: altos costos financieros, riesgos, baja rentabilidad, conflictos para ser financiado en medios permanentes y toma de decisiones, es solo ahí, cuando recurren a medios de análisis más sofisticados.

Para ello, es fundamental que los directivos empresariales utilicen la destreza en indicadores económicos y financieros y su respectiva interpretación, conduciendo a prácticas para profundizar y aplicar el análisis como base principal para la toma de decisiones. Esto sugiere, la necesidad de manejar fundamentos teóricos y técnicos en herramientas, actualmente en uso y lograr una mayor calidad de la información financiera. De allí, que el proceso de mejora en la toma de decisiones en una gestión financiera eficiente, que minimiza los impactos y alarga los periodos de estabilidad financiera desde el campo organizativo, empleando para ello, técnicas bien fundamentadas en las que es imprescindible no improvisar.

Por otra parte, Velásquez, Ulloa, Pazmiño \& Valencia (2017), definen el análisis financiero como una técnica de evaluación del comportamiento operativo de una empresa, el cuál es el diagnóstico de la situación actual y la predicción de eventos futuros; dirigido a lograr objetivos predeterminados. Este análisis se 
basa en la interpretación de acontecimientos financieros que han ocurrido durante el desarrollo del negocio empresarial, para la que se utilizan técnicas que, una vez aplicadas, conducen a la toma de decisiones correctas; también contribuye a la investigación de la capacidad de endeudamiento en la inversión de la empresa, con base en la información proporcionada por el campo financiero.

Las teorías financieras son herramientas excepcionalmente útiles con las que se puede inferir el rendimiento financiero de la empresa durante un período de tiempo; con la ayuda de estos análisis, se pueden extraer conclusiones sobre varios aspectos, como la rentabilidad de la salud financiera y la eficiencia operativa de la empresa. La salud financiera de la empresa se puede conocer con la ayuda de diferentes técnicas. Las mismas, señalan la posición de liquidez de la empresa para cumplir con sus obligaciones y solvencia a largo plazo. Estas, indican fortalezas y debilidades de la empresa.

El análisis financiero proporciona una comparaciónentreempresasouna comparación con el campo industrial, que pruebe los promedios comparando las razones de la empresa, con las de otras más competitivas. Una comparación entre empresas muestra la posición relativa a través de sus competidores. Si la comparación muestra una variación, se pueden identificar las posibles razones y si los resultados son negativos, las acciones correctivas pueden iniciarse inmediatamente y ponerlas en línea. También es útil para advertir sobre las necesidades corporativas y ayuda a la gerencia a tomar medidas correctivas. Por lo tanto, es necesario establecer cuáles son las herramientas financieras más óptimas para desarrollar un sector económico como las PYMES, sobre todo en el contexto nacional, en donde este fragmento empresarial, representa un muy importante sector económico para el país.
Identificar las principales herramientas de análisis financiero que utilizan las pequeñas y medianas empresas

HACT, Método Armonizado para las Transferencias de Efectivo. En la búsqueda de metodologías financieras que aporten a las pequeñas y medianas empresas, destaca el modelo HACT (Método Armonizado para las Transferencias de Efectivo), según Mero \& Valdez (2018), Chagolla, Campos \& González (2017) y Delgado \& Gómez (2018), es un conjunto simplificado de procedimientos y principios que rigen el desembolso de fondos y la presentación de informes de los gastos organizacionales. El enfoque HACT tiene un enfoque de gestión de riesgos dirigido a reducir costos de transacción asociados con la implementación del programa a través de: armonización de procedimientos y herramientas. Tratándose en un sistema de eliminación de un enfoque basado en el sistema de control y mejora de la capacidad para gestionar eficazmente los recursos, el Marco HACT se adoptó por primera vez en el año 2005. Este sistema de gestión de recursos es vital cuando se habla de administrar los activos.

Este modelo de análisis financiero se basa en un sistema de microevaluación, el cual es un proceso de evaluación del riesgo general del socio implementador, relacionado con la transferencia de efectivo. El propósito de este, es evaluar la capacidad de gestión financiera como: contabilidad, informes, adquisiciones, gestión de control interno, sistemas de información, etc. para determinar, la calificación de riesgo general (Mero \& Valdez (2020). La microevaluación se basa en un cuestionario y es realizada por firmas contables profesionales y clasifica la calificación de riesgo general como cualquiera de los siguientes cuatro:

Riesgo bajo: indica un sistema de gestión financiera bien desarrollado y un funcionamiento interno de sistemas de control 


\section{2}

con una baja probabilidad de impacto negativo potencial en la capacidad de ejecución de las PYMES.

Riesgo medio: indica un sistema de gestión financiera desarrollado y sistemas de control interno con una probabilidad moderada de impacto negativo en la capacidad de la empresa para ejecutar el programa de acuerdo con la asociación.

Riesgo significativo: indica un sistema de gestión financiera o control interno poco desarrollado con una probabilidad significativa de impacto negativo en la capacidad de la empresa para ejecutar el programa de acuerdo con la asociación.

Riesgo alto: indica un sistema de gestión financiera y un control interno poco desarrollados con una alta probabilidad de impacto negativo en la capacidad de la empresa para ejecutar el programa de acuerdo con la asociación.

Método VAR, Valor en riesgo. La noción de movimientos extremos en los precios de los activos está implícita en las prácticas actuales de gestión de riesgos dentro de las pequeñas y medianas empresas. Según Cadena \& Palomo (2015), la suficiencia de capital asume un umbral que clasifica los cambios observados en los factores de riesgo de mercado como extremos u ordinarios. En primer lugar, se elige una probabilidad para medir el "carácter extremo" de los eventos que pueden afectar una cartera en particular.

Detrás de este enfoque está el teorema del límite central, que produce una distribución normal asintótica para los factores de riesgo considerados. Sin embargo, si es posible obtener una distribución asintótica para todos los valores posibles de los factores de riesgo, ¿por qué no hacer lo mismo también para las observaciones extremas? la teoría de los extremos hace exactamente esto y proporciona una metodología que se puede utilizar para hacer inferencias estadísticas, utilizando un nuevo conjunto de herramientas financieras (Cadena \& Palomo 2015).

Por lo tanto, los cálculos de valor en riesgo proporcionados por esta metodología, utilizan la teoría de la distribución extrema y arrojan algunos resultados notables. Tanto los datos dentro como fuera de la muestra, manifiestan que la teoría de la distribución extrema funciona sorprendentemente bien al capturar, tanto la tasa de ocurrencia como el alcance de los eventos extremos en los mercados financieros. De hecho, en opinión de Cadena \& Palomo (2015), la teoría estadística de los extremos parece ser un enfoque más natural y sólido para los cálculos de gestión de riesgos.

Capital de trabajo. En gestión financiera se habla de capital de trabajo, cuando se dispone de todos los recursos financieros a largo plazo que la empresa necesita para poder realizar sus actividades en el corto plazo. En otras palabras, sirve como medida de la capacidad de una empresa para desarrollar aún más su negocio.

Si define capital de trabajo, como un ciclo de referencia para comparar la evolución de la salud financiera en el corto plazo. Para Yepes \& Restrepo (2016), el ciclo de capital de trabajo de una empresa es el tiempo que lleva convertir el capital de trabajo neto total (activos corrientes menos pasivos corrientes) en efectivo. Según Muyma \& Rojas (2018), las empresas suelen tratar de gestionar este ciclo vendiendo inventario rápidamente, recaudando ingresos de los clientes rápidamente y pagando las facturas lentamente para optimizar el flujo de caja.

Pues bien, este método es fundamental para calcular el circulante, si se quiere tener un control contable sobre el mismo y mantener una buena gestión financiera que es un futuro a largo plazo. El capital de trabajo tiene en 


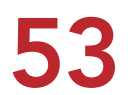

cuenta los próximos pagos y cobros, y es útil mantenerlo positivo.

Análisis de Razones Financieras (ARF): LOS índices financieros se crean con el uso de valores numéricos tomados de los estados financieros para obtener información significativa sobre una empresa. Según Acosta, Bazante \& Ojeda (2016), los números que se encuentran en los estados financieros de una empresa (balance, estado de resultados y estado de flujo de efectivo) se utilizan para realizar análisis cuantitativos y evaluar la liquidez, el apalancamiento, el crecimiento, los márgenes, la rentabilidad, las tasas de rendimiento, la valoración y más de una empresa.

Los ratios financieros se agrupan en las siguientes categorías: Ratios de liquidez, Ratios de apalancamiento, Ratios de eficiencia, Ratios de rentabilidad, Ratios de valor de mercado. Estos ratios financieros, se emplean generalmente para el seguimiento del desempeño de la empresa, por cuanto, permite la determinación de las razones financieras individuales por período y el seguimiento del cambio en sus valores a lo largo del tiempo. Se realizan para detectar tendencias de uso de recursos que puedan estar desarrollándose en una empresa. Por ejemplo, una relación deuda / activos en aumento puede indicar que una empresa está sobrecargada de deuda y que eventualmente se enfrenta al riesgo de incumplimiento.

Adicionalmente, según Acosta, Bazante \& Ojeda (2016), se emplea en las PYMES, para hacer juicios comparativos sobre el desempeño de la empresa. A través, de la comparación de las razones financieras con las de los principales competidores, se identifica si una empresa se está desempeñando mejor o peor que el promedio de la industria. Por ejemplo, comparar el rendimiento de los activos entre empresas ayuda a un analista o inversor a determinar qué empresa está haciendo el uso más eficiente de sus activos.
Técnica de Estructura Financiera (EF): En la gestión financiera, es un término significativo y es una decisión muy importante en los negocios. Según Angulo, Berrío. \& Caicedo (2014), en la estructura de capital de una empresa, en términos generales, existen principalmente dos tipos de capital, es decir, capital y deuda. De las dos, la deuda es una fuente de financiación más barata porque la tasa de interés será menor que el costo del capital y los pagos de intereses son un gasto deducible de impuestos.

La estructura de capital o apalancamiento financiero se ocupa de una cuestión de gestión financiera muy importante, el endeudamiento. En el contexto de la gestión financiera, el objetivo de cualquier decisión financiera es maximizar la riqueza del accionista o aumentar el valor de la empresa. La otra pregunta que surge en la mente en primer lugar es, si un cambio en la combinación de financiamiento tendría algún impacto en el valor de la empresa o no. La pregunta es válida ya que hay algunas teorías que creen que la combinación financiera tiene un impacto en el valor y otras creen que no tiene conexión (Angulo, Berrío. \& Caicedo, 2014).

Sea cual sea el lugar y la forma en que se obtenga la financiación, no podrá cambiar los niveles de ingresos operativos. El método de la estructura financiera, puede, como máximo, tener un impacto en los ingresos netos 0 en el EPS (beneficio por acción). Cambiar la combinación de financiamiento significa cambiar el nivel de deudas. Este cambio, en los niveles de financiación puede afectar los intereses a pagar por esa empresa. La disminución de los intereses aumentaría la utilidad neta $y$, por lo tanto, las EPS y es una creencia generalizada que el aumento de las EPS conduce a un aumento en el valor de las PYMES (Angulo, Berrío. \& Caicedo, 2014).

Contabilidad de Administración Estratégica: La Contabilidad de Gestión Estratégica (SMA), 


\section{4}

según Velásquez, Ulloa, Pazmiño \& Valencia (2017), se define como el suministro y análisis de información sobre una empresa y sus competidores para su uso en el desarrollo y seguimiento de la estrategia empresarial. Por lo tanto, se encuentra en la interfaz entre la gestión estratégica y la contabilidad. Algunos han sugerido que implica información contable que respalda la gestión estratégica (SMA) y otros que se relaciona con todas las técnicas de contabilidad de gestión que tienen una perspectiva estratégica (SMA).

Cualquiera que sea la interpretación exacta (SMA) que implique una mayor contribución de los contadores a la formulación e implementación de la estrategia, sugiere que los contadores se alejen de las preocupaciones puramente financieras hacia cuestiones comerciales más amplias, cómo, por ejemplo, su empleo en el análisis financiero de pequeñas y medianas empresas.

Algunos investigadores tienden a usar una definición estrecha, explicando SMA como una técnica centrada en la competencia, como es Bjøornenak \& Olson 1999 (citado por Velásquez, Ulloa, Pazmiño \& Valencia, 2017); mientras que otros lo ven relacionado con el posicionamiento estratégico y particularmente integrado con la contabilidad de gestión y el marketing dentro de las PYMES. En un papel influyente que implica el coste de los "atributos" del producto, se basa en la teoría de los mercados contestables, señalando la necesidad para el mantenimiento de una ventaja de costes sobre la competencia. En los últimos años esta técnica se conoce como Gestión Estratégica de Costos (SCM), que involucra consideración de la cadena de valor, el generador de Costos y el análisis de ventaja competitiva.

Enfoque de Modelado de Pronóstico: En prácticamente todas las decisiones que toman, los ejecutivos de hoy, consideran algún tipo de pronóstico. Las predicciones sólidas de las demandas y las tendencias ya no son artículos de lujo, sino una necesidad, si los gerentes quieren hacer frente a la estacionalidad, los cambios repentinos en los niveles de demanda, las maniobras de reducción de precios de la competencia, las huelgas y los grandes cambios de la economía. Según Romero (2013), los pronósticos pueden ayudarlos a lidiar con estos problemas; pero puede ayudarles más cuanto más sepan, acerca de los principios generales de la previsión, lo que puede y no puede hacer por ellos en la actualidad y las técnicas que se adaptan a sus necesidades del momento. Aquí, los autores intentan explicar el potencial de los pronósticos a los gerentes, dentro de un sector tan dinámico como las PYMES, prestando especial atención a los pronósticos de ventas para los productos, ya que estos han madurado a lo largo del ciclo de vida del producto.

En este sentido, en el sector gerencial dirigido a manejar la creciente variedad y complejidad de los problemas de pronóstico de gestión en las PYMES, han desarrollado muchas técnicas de pronóstico en los últimos años. Cada uno tiene su uso especial y se debe tener cuidado para seleccionar la técnica correcta para una aplicación en particular. Tanto el administrador como el pronosticador tienen un papel que desempeñar en la selección de técnicas; y cuanto mejor comprendan la gama de posibilidades de previsión, más probable será que los esfuerzos de previsión de una empresa den sus frutos (Romero, 2013).

La selección de un método apropiado en las PYMES, depende de muchos factores: el contexto del pronóstico, la relevancia y disponibilidad de datos históricos, el grado de precisión deseable, el período de tiempo a pronosticar, el costo / beneficio (o valor) del pronóstico para la empresa, y el tiempo disponible para realizar el análisis. 
Describir la situación financiera que presentan las pequeñas y medianas empresas

A nivel internacional. Las PYMES no pueden estar al margen del contexto económico en el que se encuentra sumergido el mundo actualmente. En este sentido, el análisis se puede abordar desde un enfoque macroeconómico centrado en el contexto como elemento determinante en sus procesos de financiamiento, especialmente los externos. En los Estados Unidos, el sobreendeudamiento de las economías y la comunidad empresarial, han llevado a las PYMES a una crisis financiera desde 2007. Aunque hay opiniones diferentes estudios, académicos sobre las causas de esta crisis (López, 2015).

Sin embargo, el origen es solo una "burbuja inmobiliaria" generada en los Estados Unidos, pero también hay que tener en cuenta una visión económica agresiva que se ha generado en ese país y el marco regulatorio, pre-establecido como causantes del periodo de crisis. No obstante, según varios analistas, señalan la existencia de una liquidez masiva generada debido a varios factores, como: la fuga de capitales, la burbuja tecnológica, escenario de fuerte alquiler, una política monetaria de carácter expansivo. Lo cual, ha llevado a un endeudamiento excesivo, dando a las empresas acceso a hipotecas que no tenían capacidad crediticia mínima para hacerlo. De hecho, el marco económico se vio impulsado por las bajas tasas de interés (1\%) que aportó mucha liquidez, buscando un destino para invertir. Esto siguió una gran expansión crediticia, en la medida que se hicieron más asequibles (López, 2015).

En el campo de las PYMES españolas, según Di Pietro, Palacín \& Roldán (2015) y Palacín $\&$ di Pietro (2016), es importante tener en cuenta el entorno institucional regional para conocer mejor las diferencias entre regiones dentro de un solo país. Además, la relación entre el factor regional, medido como nivel de desarrollo, y capital de la estructura es negativa. Este resultado, sugiere que la deuda de las PYMES es el mayor problema que presentan las mismas, lo cual se presenta con más frecuencia en los países menos desarrollados. En cuanto a regiones españolas, una posible explicación es que las regiones menos desarrolladas, que son más pobres, tienen menos recursos propios para invertir y, por lo tanto, deben depender de la deuda. Además, en las regiones desarrolladas, los factores institucionales regionales pueden favorecer el uso de la deuda.

En segundo lugar, todos los factores de adquisición de capital considerados son significativos en la explicación de las decisiones de financiamiento. Además, el sector empresarial tiene un efecto tanto directo como indirecto vía estructura de activos sobre estructura de capital. Finalmente, los factores de adquisición de capital y el sector empresarial explican mayor variación en la estructura de deuda que la dependencia regional. Siendo así, este sector económico en España se encuentra en problemas de endeudamiento severos que impiden la maniobrabilidad y minimizan su capacidad de generación de empleo, una vez que los empresarios optan por, cada vez, contar con menores empleados (Arimany, Sabata \& Viladecans, 2017).

Además, según Grimaldi, García, Gómez \& Gonzalo (2016), se puede afirmar que la crisis económica ha afectado a las PYMES, de forma diferente según el tamaño de cada uno. El resultado positivo de algunas en el segundo periodo después de la crisis económica latinoamericana, constata que las medianas empresas son más eficientes en el uso de sus recursos económicos o en la obtención de beneficios de sus activos, obteniendo mejores resultados, en términos de valores medios de capital. Mientras que las grandes, pequeñas y microempresas muestran valores medios inferiores, encontrándose en decrecimiento a 


\section{6}

juzgar por los índices de operatividad, lo que reflejan menor desarrollo y un decrecimiento en cerca de un $30 \%$ en creación de PYMES.

A nivel nacional: En Colombia, los aspectos financieros también reflejan una serie de limitaciones para las PYMES; por ejemplo, son muy pocas las empresas que han establecido políticas de liquidez y rentabilidad o rara vez lo permiten. Según Barbosa, Camelo, Deaza \& Rodríguez (2018), estas presentan problemas financieros, debido a gestiones empresariales inadecuadas, especialmente en los aspectos financieros.

Este estudio, identificó grupos de empresas, cada uno formado con características específicas en los aspectos comerciales y financieros, concluyendo, que estas, en su mayoría carecen de planificación financiera y poca experiencia en exportación y un pequeño grupo poseen experiencia relevante en mercados internacionales; ya que cerca de un $70 \%$ de las PYMES están orientadas a la exportación, fracasan antes de los 5 años, según cifras del Ministerio de Comercio Exterior (citado por Barbosa, Camelo, Deaza \& Rodríguez (2018).

En este sentido, existe un grado de preparación hacia las empresas para exportar, que deben complementarse y medirse con estrategias de financiamiento coordinado y una evaluación más profunda de los mercados internacionales; como parte de los factores internos que, acompañado de iniciativas gubernamentales generadas para apoyar a las empresas en sus procesos de internacionalización y promoción en su desarrollo organizacional, prevén, un proceso de internacionalización más amplio y eficiente en los PYMES. A partir de entonces, se puede afirmar que las PYMES en Colombia se encuentran en un claro proceso de decrecimiento (Barbosa, Camelo, Deaza \& Rodríguez, 2018).
Por otra parte, el análisis de fallas comerciales presentes en las PYMES, se basa en la metodología descrita por Romero (2013), examinando la mayor confiabilidad de la probabilidad condicional. En ese sentido, en Colombia, las empresas fallidas, están formada por 1.312 organizaciones que se encuentran en situación de liquidación judicial, concordato, reestructuración y reorganización para el período 2005-2011. En segundo lugar, se determinan que actualmente las causas que llevan a la quiebra de las pequeñas y medianas empresas en Colombia son: Pasivo a largo plazo / Activo total, Pasivo total (Activo corriente-Inventario) / Activo total. Estas variables financieras en las pequeñas y medianas empresas que fracasan se enumeran a través de un mayor grado de apalancamiento a largo plazo y baja concentración de efectivo en relación con los activos totales, que no son suficientes para cubrir los pagos de la deuda. Por tanto, los problemas prácticos en la actualidad, son la liquidez de la deuda, coherentes con las causas de insolvencia descubiertas por la Superintendencia de Sociedades de Colombia 2012 (citado por Angulo, Berrio \& Caicedo, 2014) que señalan el alto endeudamiento y reducción de ventas (causando falta de liquidez) como señales de identidad del fracaso empresarial.

Uno de los resultados más relevantes, a nivel nacional, es que la mayor parte de las pequeñas y medianas empresas, el $60 \%$, (de cerca de 700.000 empresas), se establecen como personas jurídicas, pero existen, cerca de un 40\% (300.000 empresas) que no son formales, de ahí la importancia de implementar estrategias de formalización y posteriormente de planificación financiera. Por supuesto, la organización también se ve como un todo (Villacrés, Rodríguez \& Sánchez, 2020) en el que fallan en su totalidad los componentes financieros de las PYMES. Además, se destaca el plazo de inventario, extremadamente alto para el tipo de negocio, producido por la composición de los activos y el nivel de 
endeudamiento a corto plazo, debido a que la solvencia de estas empresas se considera bajo y la rentabilidad de los activos es muy baja, por el componente mayoritario (actual) y los efectos inflacionarios. El alto componente de endeudamiento con terceros pone a las empresas con riesgo financiero, principalmente por escasa liquidez, asumiendo compromisos de gestión de inventario ineficiente a corto plazo por la exposición a la inflación.

Por otro lado, el $65 \%$ de las empresas considera que la contabilidad es más importante que la inversión en presupuestos de proyecciones; así como la toma de decisiones que no se basa en una adecuada gestión administrativa. Es más importante la experiencia para el $45 \%$ de las empresas, también depende de la tendencia del mercado en el $20 \%$, lo que afecta el riesgo de endeudamiento sin capacidad. Adicional a ello, la importancia de la gestión financiera para las PYMES, está en último lugar con 10\% (Villacrés, Rodríguez \& Sánchez, 2020).

\section{Proponer herramientas que fortalezcan el análisis financiero en las pequeñas y medianas empresas.}

El análisis en discusión de los resultados de la investigación sobre estrategias financieras en las micro, pequeñas y medianas empresas comerciales son variados y dependen mucho del sector comercial al que se dediquen las PYMES. Por lo tanto, según Angulo, Berrío. \& Caicedo (2014), en primer lugar, es posible establecer, que debido a que el ciclo de conversión de efectivo es alto, lo que indica una mala gestión del factor crediticio, se hace necesario, reducir el uso de la técnica de apalancamiento y aumentar el método de Armonizado para las Transferencias de Efectivo, que según Mero \& Valdez (2020), proporciona conjunto simplificado de procedimientos y principios que rigen el desembolso de fondos y la presentación de informes de los gastos organizacionales, con el fin de reducir las brechas que las deudas imprimen a este sector actualmente.

Por otra parte, debido a la situación de riesgo frente a las condiciones de cambios políticos, sociales y económicos del país, es imperativa la optimización de los recursos y capacidades internas y externas, para una buena administración y ejecución de sus estrategias financieras dirigidas a la sostenibilidad y crecimiento; lo cual coloca la estrategia de capital de trabajo, como una herramienta eficiente para solventar la actual situación de crisis en la que se encuentra este sector, no solo a nivel nacional sino internacional (Muyma \& Rojas, 2018).

Asimismo, a partir de los problemas evidenciados al encontrarse poca disposición por parte de las PYMES para aplicar proceso de planificación financiera, se deben fomentar el uso de herramientas de análisis que permita desarrollar mejores capacidades de crecimiento; sobre todo tomando en consideración las cifras de decrecimiento económico y el porcentaje de supervivencia no mayor de 5 años en un número importante de empresas. Se hace imperativo, según Álvarez, Toros \& Mancini (2016), que las PYMES se apoyen en la metodología del Presupuesto Operativo (PO), el cual es un instrumento básico para esta planificación, porque, a partir de una mejor combinación posible entre precios, cantidades a vender y costos a incurrir, se consolida como una estrategia que preserva el poder de efectivo de las PYMES.

La estrategia consiste en el uso de una herramienta gerencial para planificar el flujo de efectivo de forma anticipada, teniendo en cuenta las proyecciones de ventas, menor nivel de inventarios y para reducir los costos unitarios de fabricación. Una de las utilidades de esta herramienta, es la planificación desde una mirada integral a todas las actividades de la empresa durante un ciclo completo, especialmente cuando se trata de actividades 


\section{8}

con un alto grado de estacionalidad, como es el caso de las PYMES.

Las PYMES, según López (2014), como todos los elementos del tejido económico e industrial, han sufrido las consecuencias de la crisis financiera. Su estructura y las condiciones financieras también han cambiado en los últimos años y si bien el autofinanciamiento y el financiamiento bancario siguen siendo pilares fundamentales para su financiamiento, es necesario incluir fuentes, que vayan en consonancia con algunas tendencias, prácticas y uso internacional, buscando alternativas para emprender proyectos que no son centrados en mecanismos tradicionales.

Todo esto, permite fomentar las perspectivas de consolidación del nuevo ciclo económico. Los cuales, pueden permitir a las PYMES desarrollar nuevos modelos consolidados en proyectos permanentes, que generan empleo y contribuyen al crecimiento nacional (López, 2014). Asimismo, Romero (2013), indica que las estrategias dentro de las PYMES, deben considerar estudios específicos a través de áreas completas del mercado, empleando el Modelo Explicativo del Fracaso, dadas las características especiales de los centros de negocios de Colombia. El cual, es un modelo que incluye variables categóricas de sector, tamaño y antigüedad. Los mismos, se ha centrado principalmente en las variables de carácter económico y financiero obtenidos de las instituciones financieras; para ello, es importante presentar otras variables de carácter cualitativo que pueden aportar información relevante que explique el comportamiento financiero de las PYMES.

Además, se avanza en el uso de otros métodos de estimación que consideran otros datos originales menores; entre estas metodologías se encuentran las técnicas de modelos no paramétricos y especiales de inteligencia artificial, redes, árboles de decisión, neuronales, entre otros, que podrían permitir obtener mejores índices de clasificación en variables explicativas con mayor ajustamiento, en el campo de las medianas empresas. Igualmente, se deben implementar herramientas de análisis financiero más específicas, que vayan más allá del uso de indicadores de gestión, para que se puedan proveer riesgos de pérdida en el segmento de las microempresas en Colombia. Es preocupante el número de quiebras corporativas que se relacionan con el abandono de las iniciativas estratégicas, endeudamiento masivo y debilitamiento del efectivo, que es muy característico en las PYMES (Romero, 2013).

\section{Conclusiones}

Al hacer la revisión de la literatura más reciente, se identificaron las principales herramientas de análisis financiero que utilizan las pequeñas y medianas empresas, donde destacan el HACT- Método Armonizado para las Transferencias de Efectivo, el Método VAR - Valor en riesgo, el análisis de capital de trabajo, el Análisis de Razones Financieras (ARF), la Técnica de Estructura Financiera (EF), la Contabilidad de Administración Estratégica y el Enfoque de Modelado de Pronóstico.

La situación financiera que presentan las pequeñas y medianas empresas se caracteriza por dificultades para obtener capital de trabajo que les representa riesgo de quiebra en el mediano y largo plazo. Por lo tanto, se identificó, tanto a nivel nacional como internacional, dificultades como altos volúmenes de deudas que superan la capacidad de pago, la falta de estrategias financieras y es escaso interés por la planificación de los recursos, ya que se basan más en estimaciones subjetivas de las tendencias del mercado y en la experiencia que tienen en el sector económico donde se desempeñan.

Se sugieren los métodos de análisis financiero como el método de Armonizado 
para las Transferencias de Efectivo, que permite reducir las brechas de endeudamiento; adicional a ello, con el fin de aumentar su rentabilidad, se sugiere el empleo del modelo de capital de trabajo para analizar integralmente los recursos involucrados en la producción de las PYMES y solventar las necesidades de efectivos. Por otra parte, estas empresas se deben apoyar en la metodología del Presupuesto Operativo (PO), el cual es un instrumento básico para la planificación, con el fin de mejorar la combinación posible entre precios, cantidades a vender y costos a incurrir, lo que permite cierta anticipación a los posibles resultados, para reducir la incertidumbre y tomar acciones oportunas en la gestión de los recursos financieros.

\section{Referencias}

Acosta, M., Bazante, Y. \& Ojeda, Y. (2016). Factores que inciden en la toma de decisiones financieras de las pymes del sector construcción, Popayán, 2016. Revista Libre Empresa, 14(1), 147-165

Álvarez, J., Toros, C. \& Mancini, M. (2016). Evaluación económica- financiera de pequeños proyectos en hotelería. Revista realidad, tendencias y desafíos en turismo, 16(14), 98106

Angulo, F., Berrío, H. \& Caicedo, L. (2014). Estrategias de inversión en capital de trabajo aplicadas por las micros, pequeñas y medianas empresas colombianas de comercio textil en el municipio de Maicao. Revista Dimensión Empresarial, 12(2), 69-82

Arimany, N., Sabata, A. \& Viladecans, C. (2017). Un análisis de la estructura financiera de las pymes de una comarca en periodo de crisis: el caso de Osona. RIGC, 15(29). 2-16

Barbosa, M., Camelo, M., Deaza, J. \& Rodríguez, G. (2018). Diagnóstico financiero y desempeño exportador de las MIPYMES colombianas.
Panorama Económico, $26(4$ ), 435-458

Cadena, J. \& Palomo, R. (2015). Análisis de la información económico-financiera: la utilización de los métodos lineales de pronóstico en las empresas de Bogotá. Universidad CeuSan Pablo, Bogotá, Colombia

Chagolla, F. M., Campos, D. P., \& González, S. F. (2017). El presupuesto de efectivo en las Pymes. Ciencias Empresariales, 28-62

Delgado, G. I., \& Gómez Chi, S. V. (2018). Importancia de la contabilidad dentro de las Pymes. Contribuciones a la economía, 1-12

Di Pietro, F., Palacín, M. \& Roldán, J. (2015). Desarrollo regional y estructura de capital de las PYME. Cuadernos de Gestión, 18(1), 37-60

Grimaldi, M., García, J., Gómez, R. \& Gonzalo, B. (2016). Impacto de la crisis económica en la rentabilidad de las empresas de gestión de instalaciones de ocio deportivo según tamaño, un estudio empírico. Revista de Psicología del Deporte, 25(1), 51-54

López, J. (2014). Evolución y análisis de la financiación de la PYME en España. Financiación empresarial, 6 (879), 1-10

Martínez, C. (2014). Gestión financiera del circulante y creación de valor en la empresa. Universidad de Murcia, Murcia, España

Mero, J. \& Valdez, D. (2018). Análisis de la aplicabilidad de herramientas para la administración del capital de trabajo en las PYMES comerciales del cantón Chone. Polo del conocimiento, 48(5), 634-652

Muyma, A. \& Rojas, D. (2018). Estrategias de gestión de efectivo y su relación con la rentabilidad en PYMES ecuatorianas: sectores comercio y manufactura. Digital Publishem $4(51), 5-22$ 
60

Palacín-Sánchez, M.J \& di Pietro, F. (2016). The role of the regional financial sector in the capital structure of small and medium-sized enterprises (SMEs). Regional Studies, 70 (7), 1232-1247. DOI. 10.1080/00343404.2014.1000290

Pérez, D. (2018). El capital humano como generador de valor financiero en las PYMES. Centro de investigaciones y ciencias gerenciales, 18(1), 1858-1963

Romero, F. (2013). Variables financieras determinantes del fracaso empresarial para la pequeña y mediana empresa en Colombia: análisis bajo modelo Logit. Pensamiento \& gestión, 34(5), 235-277

Velásquez, B., Ulloa, C., Pazmiño, E. \& Valencia, M. (2017). Estrategias financieras aplicadas al proceso de sostenibilidad y crecimiento de las pymes del sector comercial. Revista Publicando, 4(2), 16-33

Villacrés, J., Rodríguez, O. \& Sánchez, D. (2018). Gestión administrativa y financiera, perspectiva desde las pequeñas y medianas empresas de la zona centro de la ciudad de Riobamba. Polo del conocimiento, 5(2), 179-199

Yepes, D., \& Restrepo, D. (2016). Determinantes del nivel de efectivo de las compañías colombianas. Lecturas de Economía, 85(85), 243-276. https:// doi. org/10.17533/udea.le.n85a08 